\title{
The pre-ejection period during the Muller manoeuvre: mismatch or misreading?
}

\author{
Karim Bendjelid
}

Published online: 8 December 2013

(C) Springer Science+Business Media New York 2013

The greatest obstacle to discovery is not ignorance, it is the illusion of knowledge

Daniel J. Boorstin (1914-2004)

In this issue of the Journal of Clinical Monitoring and Computing, Dr. Vistisen and colleagues emphasise the inadequacy of the variation in the pre-ejection period (PEP) following deep breathing to predict the hemodynamic response to controlled haemorrhage [1]. The present clinical report may lead to the implication that this marker of hypovolemia should be disregarded in cases of spontaneous ventilation.

The PEP includes excitation-contraction coupling and isovolumic contraction. Accordingly, the normovolemic left ventricle has a short PEP, whereas a hypovolemic ventricle has a long PEP. In this regard, mechanistically, searching the preload prediction from the pre-ejection period measurement makes sense. Indeed, there have been numerous published studies, both in human patients and in various animal models supporting the use of the pre-ejection period to characterise the preload [2]. These findings are based on direct observations and are independent of the type of respiration, as studies have been performed under conditions of spontaneous [3] and mechanical ventilation [4, 5].

With this in mind, it is rather surprising that little work has been done to measure the reliability of these systolic time intervals during deep inspiration and/or the Muller manoeuvre [6]. The article by Vistisen et al. addresses this

This comment refers to Simon Tilma Vistisen et al. available at doi: 10.1007/s 10877-013-9526-6.

K. Bendjelid ( $\square)$

Intensive Care Division, Geneva University Hospitals,

1211 Geneva 14, Switzerland

e-mail: karim.bendjelid@hcuge.ch issue by presuming that through its impact on the respiratory change in PEP $(\triangle \mathrm{PEP})$, a deep breathing manoeuvre could be a preload varying method useful for predicting the magnitude of change in the cardiac output (CO) following blood donation in healthy volunteers. Interestingly, it might seem strange that the authors found that it was impossible to demonstrate such a predictive value. So far, the hypothesised underlying physiology was confirmed, as whatever the deep breathing frequency $(0.1$ vs. $0.167 \mathrm{~Hz})$, the manoeuvre induces characteristic fluctuations in the PEP pattern. However, the authors demonstrated that because the PEP is a parameter that is strongly dependent on heart rate variability (HRV), the $\triangle \mathrm{PEP}$ also depends on the respiratory induced HRV (RMSSD).

On a first reading, there are several open questions arising from the study of Dr. Vistisen et al.; perhaps the most important is the reason why the cyclic change in the PEP ( $\triangle \mathrm{PEP})$ was not able to predict the stroke volume responses to controlled haemorrhage. Indeed, it might seem strange that at the same time, the authors demonstrate that whatever the deep breathing frequency $(0.1$ vs. $0.167 \mathrm{~Hz})$, this manoeuvre induces significant characteristic fluctuations in the PEP values. Although, we may speculate that a noisy pulse plethysmographic signal may make an accurate assessment of the $\triangle \mathrm{PEP}$ difficult [7]; in this regard the methodology used by the authors is perfect.

In principle, the absence of the clinical relevance of cyclic changes in the PEP while the same parameter is sensitive to a deep inspiratory manoeuvre may result from the influence of the expiratory phase on the PEP measurements [8]. Indeed, a forced inspiratory manoeuvre may be followed by a forced expiratory effort that increases the left-ventricular afterload through an increase in the abdominal pressure. Taking everything into consideration, 
the present deep expiration may limit the swing of the left ventricular stroke volume and its impact on the $\triangle \mathrm{PEP}[8$, 11]. In reality, to my knowledge, there are different hemodynamic "schools" when the physiology of deep breathing is studied, as several research papers have demonstrated that standardisation is very difficult to assess $[9,10]$. For instance, a deep inspiration both decreases the intrathoracic pressure and increases the intra-abdominal pressure, changing at the same time the stressed and unstressed blood volume, venous return, cardiac preload, contractility and afterload [11]. Such a standard preload varying method may not really exist for humans, although several groups have used the Muller and Valsava manoeuvres to assess cardiovascular functions [12, 13]. In fact, there is evidence that it is very difficult to match a deep exhalation with a deep expiration in the same way.

One additional consideration in assessing the study by Vistisen et al. is that the authors sound an important note of caution when interpreting such data. For instance, they also acknowledge that healthy persons have an ability to compensate for moderate blood removal through various physiological mechanisms. As they note, this has serious implications when such measurements are determining the clinical utility and application of a manoeuvre that is potentially able to assess the circulatory state. To broaden this point, there is also evidence that pulsus paradoxus affects the impact of the Muller manoeuvre on the circulatory state $[13,14]$, and in this regard, the authors should have excluded all the patients with a right ventricular bundle branch.

In short, the message from this study is the lack of the ability of the $\triangle$ PEP measurements to assess a preload dependency state and to guide fluid therapy in spontaneously breathing patients. However, in my view, the present results should be interpreted with caution as various methodological improvements could significantly increase the reliability of this approach combining deep breath and systolic time interval measurements. Indeed, when taking the RMSSD into account, it could be that the present manoeuvre needs to be performed with simply one breath.
These findings could have important implications for understanding the effects of cardiopulmonary interaction manoeuvres and their potential to eventually become the most valuable markers of preload dependency in spontaneously breathing patients.

\section{References}

1. Vistisen et al. J Clin Monit Comput. 2014 (in press).

2. Weissler AM. Current concepts in cardiology. Systolic-time intervals. N Engl J Med. 1977;296(6):321-4.

3. Buch J, Egeblad H, Hansen PB, Kjaergard H, Waldorff S, Steiness E. Correlation between changes in systolic time intervals and left. Br Heart J. 1980;44(6):668-71.

4. Bendjelid K, Suter PM, Romand JA. The respiratory change in preejection period: a new method to predict fluid responsiveness. J Appl Physiol. 2004;96(1):337-42.

5. Brundin T, Hedenstierna G, McCarthy G. Effect of intermittent positive pressure ventilation on cardiac systolic time intervals. Acta Anaesthesiol Scand. 1976;20(4):278-84.

6. Weissler AM, Harris WS, Schoenfeld CD. Bedside technics for the evaluation of ventricular function in man. Am J Cardiol. 1969;23(4):577-83.

7. Bendjelid K. The pulse oximetry plethysmographic curve revisited. Curr Opin Crit Care. 2008;14(3):348-53.

8. Soubrier S, Saulnier F, Hubert H, Delour P, Lenci H, Onimus T, et al. Can dynamic indicators help the prediction of fluid responsiveness in spontaneously breathing critically ill patients? Intensive Care Med. 2007;33(7):1117-24.

9. Capel LH, Smart J. The forced expiratory volume after exercise, forced inspiration, and the Valsalva and Muller manoeuvres. Thorax. 1959;14:161-5.

10. Morgan BC, Dillard DH, Guntheroth WG. Effect of cardiac and respiratory cycle on pulmonary vein flow, pressure, and diameter. J Appl Physiol. 1966;21(4):1276-80.

11. Buda AJ, Pinsky MR, Ingels NB Jr, Daughters GTD, Stinson EB, Alderman EL. Effect of intrathoracic pressure on left ventricular performance. N Engl J Med. 1979;301(9):453-9.

12. Bake B. Distribution of pulmonary blood flow during the Valsalva and Muller maneuvers. Scand J Clin Lab Invest. 1971;27(4):307-14.

13. Massumi RA, Mason DT, Vera Z, Zelis R, Otero J, Amsterdam EA. Reversed pulsus paradoxus. N Engl J Med. 1973;289(24):1272-5.

14. McGregor M. Current concepts: pulsus paradoxus. N Engl J Med. 1979;301(9):480-2. 